\title{
Risk assessment tools for QT prolonging pharmacotherapy in older adults: a systematic review
}

\author{
Simone Skullbacka ${ }^{1} \cdot$ Marja Airaksinen $^{1}$ (i) $\cdot$ Juha Puustinen ${ }^{2,3}$ (D) $\cdot$ Terhi Toivo $^{3,4}$ (i)
}

Received: 2 April 2021 / Accepted: 25 January 2022 / Published online: 14 February 2022

(c) The Author(s) 2022

\begin{abstract}
Purpose Many drugs are associated with the risk of QT prolongation and torsades de pointes (TdP), and different risk assessment tools (RATs) are developed to help clinicians to manage related risk. The aim of this systematic review was to summarize the evidence of different RATs for QT prolonging pharmacotherapy.

Methods A systematic review was conducted using PubMed and Scopus databases. Studies concerning risk assessment tools for QT prolonging pharmacotherapy, including older adults, were included. Screening and selection of the studies, data extraction, and risk of bias assessment were undertaken.

Results A total of 21 studies were included, involving different risk assessment tools. Most commonly used tools were risk scores $(n=9)$, computerized physician order entry systems $(n=3)$, and clinical decision support systems $(n=6)$. The tools were developed mainly for physicians and pharmacists. Risk scores included a high number of risk factors, both pharmacological and non-pharmacological, for QT prolongation and TdP. The inclusion of patients' risk factors in computerized physician order entry and clinical decision support systems varied.

Conclusion Most of the risk assessment tools for QT prolonging pharmacotherapy give a comprehensive overview of patient-specific risks of QT prolongation and TdP and reduce modifiable risk factors and actual events. The risk assessment tools could be better adapted to different health information systems to help in clinical decision-making. Further studies on clinical validation of risk assessment tools with randomized controlled trials are needed.
\end{abstract}

Keywords Risk assessment tools $\cdot$ QT prolongation $\cdot$ Torsades de pointes $\cdot$ Older adults $\cdot$ Risk management

Terhi Toivo

terhi.toivo@helsinki.fi

Simone Skullbacka

simone.skullbacka@gmail.com

Marja Airaksinen

marja.airaksinen@helsinki.fi; marjaairaksinen@gmail.com

Juha Puustinen

juha.puustinen@helsinki.fi

1 Clinical Pharmacy Group, Division of Pharmacology and Pharmacotherapy, Faculty of Pharmacy,

University of Helsinki, Viikinkaari 5 E, P.O. Box 56, 00014 Helsinki Helsinki, Finland

2 Unit of Neurology, Satasairaala Central Hospital, Satakunta Hospital District, Pori, Finland

3 Clinical Pharmacy Group, Division of Pharmacology and Pharmacotherapy, Faculty of Pharmacy, University of Helsinki, Viikinkaari 5 E, P.O. Box 56, 00014 Helsinki, Finland

4 Hospital Pharmacy, Tampere University Hospital, Pirkanmaa Hospital District, Tampere, Finland

\section{Introduction}

Many drugs are associated with the risk of QT prolongation and torsades de pointes (TdP) [1]. TdP is a rare polymorphic ventricular tachycardia that can cause reversible syncope, ventricular fibrillation, and death [2,3]. According to the QTDrugs Lists of CredibleMeds, about 200 drugs are associated with the risk of QT prolongation and/or cause TdP [1]. Based on ongoing and systematic analysis of available evidence, drugs may be placed into four QTDrugs Lists on the CredibleMeds website [1]. List 1 contains drugs that prolong the QT interval and are known for their risk of TdP, even when taken as recommended [1].

Several risk factors for QT prolongation and TdP are known [3, 4]. QT prolonging drugs were responsible for $48 \%$ of the cases of QT prolongation in a Canadian study [5]. Several non-pharmacological factors, including female sex and age $\geq 65$ years, several diseases, as well as electrolyte disturbances were found as risk factors in a quite recent 
systematic review [4]. Patients with multiple clinically recognizable risk factors, like older adults with polypharmacy and comorbidities, have an increased risk for TdP $[6,7]$.

Concomitant use of QT prolonging drugs is common and has increased in in- and outpatients [8-10]. When contemplating pharmacotherapy with a QT prolonging drug, patient-specific risk factors need to be considered $[6,11]$. However, the ECG screening rate of emergency department patients receiving QT prolonging drugs is considered low (20.9\%) [9]. The American Heart Association and the American College of Cardiology Foundation have released recommendations on the prevention and management of QT prolonging pharmacotherapy and drug-induced TdP, mainly concerning ECG monitoring in an inpatient setting rather than other strategies that may identify patients at higher risk $[6,12]$. The QT interval corrected for heart rate $(\mathrm{QTc})$ is an indicator of TdP risk, but it does not fully account for all the risk by itself $[6,11,13]$. Use of a risk assessment tool (RAT) may be a more effective way to identify patients at risk of QT prolongation and TdP for whom repetitive or continuous ECG monitoring, discontinuation of QT prolonging drugs, or serum electrolyte concentration monitoring may be necessary $[12,14]$.

The aim of this review was to systematically summarize the evidence of different RATs for QT prolonging pharmacotherapy.

\section{Material and methods}

\section{Data sources and retrieval of material}

This systematic review was conducted according to the PRISMA guidelines [15, 16]. PubMed and Scopus databases were searched for potential studies from 2005 to 2017 (Supplementary Table S1). Author SS worked in a research team helping in the search process (author TT and an information specialist at the Medical Library, University of Helsinki). In PubMed, MeSH (Medical Subject Headings) terms were used and all fields were searched. In Scopus, the fields' title, abstract, and keywords were searched. To avoid searching for duplicates in Scopus, the search was filtered with "AND NOT INDEX (MEDLINE)." The literature search began on September 22, 2017. Alerts on new articles from the databases were sent to the author's (SS) e-mail. The search was updated in August 9, 2021.

\section{Inclusion and exclusion criteria}

Any study design, excluding narrative reviews, was included. The RAT for QT prolonging pharmacotherapy could be used by any healthcare professional, in any healthcare settings. The RAT could involve anything from a risk score calculator to using computer software or databases to assess the risk. Studies involving risk assessment without using a tool or studies only involving ECG measurement and QT correction were excluded. The studies were included if they involved older adults ( $\geq 65$-year-olds [17]) or the mean/median age of the study participants was $\geq 65$ years. Articles were included if written in English and if full-text was available through the University of Helsinki Library.

\section{Selection of studies}

Titles and abstracts of studies were read by author SS/TT. In cases of unclear articles, other authors also read the articles. Reference lists of included studies were reviewed. Studies were selected based on title and the article abstract. Full texts were retrieved for selected studies.

\section{Quality assessment of included studies}

The included studies were assessed for strengths and limitations by considering the Grading of Recommendations Assessment, Development, and Evaluation (GRADE) approach [18]. The study design was considered for each included study and authors estimated the evidence level and risk of bias, if possible.

\section{Data extraction and analysis}

Full-text articles were read (SS/TT), and the following data were extracted and analyzed qualitatively: country; study design, and setting; cohort or sample; number of patients; definition of long QT interval; QT correction formula used; description of the RAT used, strengths and limitations of the RAT, and the study and the primary user of the RAT. The studies were categorized and analyzed according to the RAT used. A detailed summary table of the included studies was made (Supplementary Table S2).

\section{Results}

\section{Study selection and description of included studies}

The PRISMA diagram of literature search and inclusion process is shown in Fig. 1 [16]. In total, 21 studies were included in the systematic review in which 11 different RATs for prolonged QT were used [12, 14, 19-24, 31-33, 36, 40, $43,44]$. Most commonly used tools were risk scores $(n=9)$ [12, 14, 21-27], computerized physician order entry systems $(n=3)$ [31-33], and clinical decision support systems $(n=6)$ $[19,20,36-39]$. Of the studies, eight were conducted in the USA [12, 21, 22, 32, 33, 36, 38, 44], four in Belgium [14, 23 , 24, 27], two in Australia [25, 40, 43], one in Germany [20], 
Fig. 1 PRISMA diagram of literature search and inclusion process. PRISMA, preferred reporting items for systematic reviews and meta-analysis
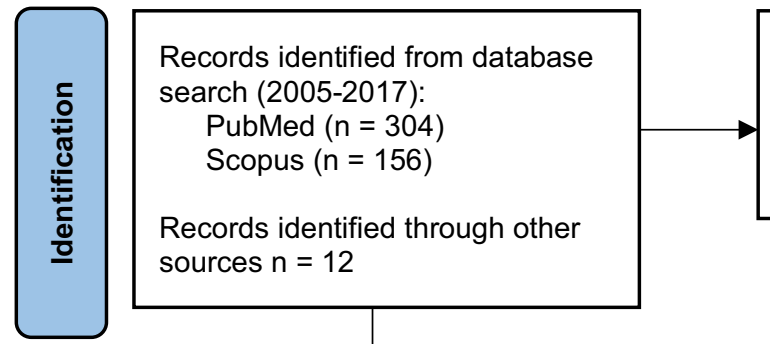

Records removed before

screening:

Duplicate records removed $(n=2)$

Records identified through other sources $n=12$

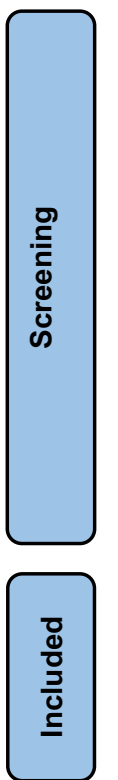

\begin{tabular}{|c|c|}
\hline $\begin{array}{l}\text { Records screened through } \\
\text { reading title and abstract } \\
(\mathrm{n}=470)\end{array}$ & $\begin{array}{l}\text { Records excluded } \\
\text { Did not fulfil inclusion criteria } \\
(n=403) \\
\text { Article not written in English } \\
(n=25)\end{array}$ \\
\hline $\begin{array}{l}\text { Reports sought for full-text } \\
\text { retrieval } \\
(n=42)\end{array}$ & $\begin{array}{l}\text { Reports not retrieved } \\
(\mathrm{n}=3)\end{array}$ \\
\hline
\end{tabular}

Reports assessed for eligibility $(\mathrm{n}=39)$

Full-text retrieved from another source $(n=1)$

Reports excluded:

Did not fulfil inclusion criteria $(n=25)$

Studies included in review $(n=15)$

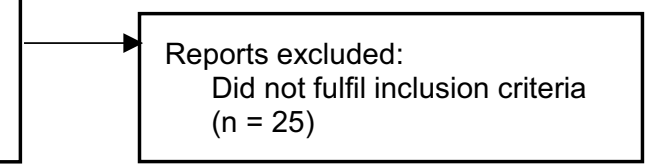

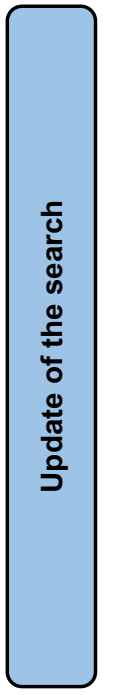

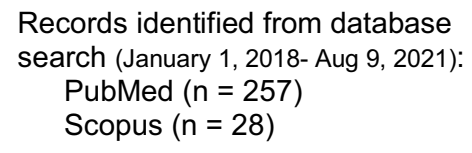

Records identified from database search (January 1, 2018- Aug 9, 2021): PubMed $(n=257)$ Scopus $(n=28)$

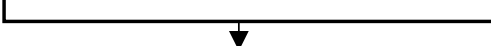

Records screened through reading title and abstract $(n=285)$

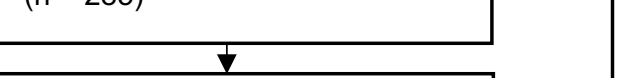

Records excluded

Did not fulfil inclusion criteria ( $\mathrm{n}=259$ )

Article not written in English $(n=1)$

Article not retrieved $(n=1)$

Reports sought for full-text retrieval

$(n=24)$

Reports excluded:

Did not fulfil inclusion criteria ( $n=18)$

Studies included in the updated review $(n=6)$

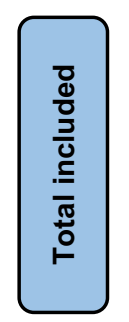

Studies included in review Total $n=15+6=21$ 
four in the Netherlands [26, 31, 37, 39], and one in Sweden [19]. The RATs were developed mainly for physicians and pharmacists. Most of the studies were conducted in inpatient settings. Of the studies, one applied a prospective controlled interventional study design [20], while the majority were observational studies (Supplementary Table S2).

\section{Comparison of studies including risk scores $(n=9)$}

Nine studies described a risk score for QT prolongation as a RAT (Table 1; Supplementary Table S2) [12, 14, 21-27]. In all nine studies, ECG data were available [12, 14, 21-27]. The definition of long QT interval differed slightly between the studies (Table 1). The QT interval was corrected for heart rate with three different correction formulae, Bazett's [28], Fridericia's [29], and Rautaharju's correction formulae [30] (Table 1).

In addition to QT prolonging drugs, the risk scores included non-pharmacological risk factors for QT prolongation such as demographic factors, QT interval affecting clinical conditions, and morbidities and electrolyte disturbances (Table 1). QT prolonging drugs were mainly identified using the QTDrugs Lists of CredibleMeds [1, 12, 14, 21-27]. Tisdale et al. [12] stated that evidence of QT prolonging drugs was taken from published trials and case reports, and CredibleMeds [1] was a used source. Included QT interval affecting diagnoses differed between the risk scores used in the studies (Table 1). The original RISQ-PATH score by Vandael et al. [14, 24] contained the highest number of risk factors, obtained from a systematic review [4], and the least number of co-morbidities was found in the risk score by Tisdale et al. [12]. The RISQ-PATH score also included risk factors that have not been validated as risk factors (hypertension, cigarette smoking, obesity, and others).

A limitation of five of the studies [21-25] was the lack of information on predictive performance (Table 1). The predictive performance was presented in four studies (Table 1) [12, 14, 26, 27]. In the study on the pro-QTc score, an institution-wide QT alert system for assessment of mortality was developed [21]. The system screened all ECGs performed and alerted physicians if finding QTc $\geq 500 \mathrm{~ms}$. The risk scores by Vandael et al. [14, 23, 24, 27] were developed in various hospital wards with different specialties, which makes them more generalizable than the risk score by Tisdale et al. [12], developed in cardiac critical care units. Selection bias may be present in some of the studies [12, 21-23]. The RISQ-PATH score developed by Vandael et al. [14] consists of many predictors that are frequently not available, which may challenge the use in clinical practice. The risk of QT prolongation or TdP may have been underestimated in the studies including the preliminary RISQ-PATH score due to missing lab results, exclusion of patients, or unrecognized TdP cases because of lack of information in the patient files or wrongly coded cases [14, 24]. In a later study by Vandael et al. [27], the RISQ-PATH score was optimized and validated in a large patient cohort, and an algorithm was proposed to be used in clinical decision support systems to create smart QT alerts. Risk factors excluded from the original RISQ-PATH score [14] were diabetes, number of drugs in list 2 of CredibleMeds, and thyroid disturbances.

Bindraban et al. [26] developed and validated two risk models (complete and simplified), both having a lower number of risk factors than the RISQ-PATH score [14]. Risk models used variables that usually are automatically available in healthcare information systems, and therefore, the risk models could be implementable in a clinical decision support system.

\section{Comparison of studies on computerized physician order entry systems $(n=3)$}

Computerized physician order entry systems (CPOE), used to reduce the risk of QT prolongation and related morbidities, were studied by van der Sijs et al. [31], Muzyk et al. [32], and Sorita et al. [33] (Table 2; Supplementary Table S2). The studies did not measure outcomes such as occurrence of cardiovascular illness, TdP, or mortality. The retrospective studies [31,32] used small sample sizes; thus, their generalizability is limited and selection bias probable. The quasi-experimental study [33] was conducted in an institution-wide setting and could not exclude confounding factors and dependency of data due to the study design [33]. The CPOE QT alert override rate was $77 \%$.

\section{Comparison of studies on clinical decision support systems $(n=6)$}

Clinical decision support systems (CDSS) were studied in six studies [19, 20, 36-39] (Table 3; Supplementary Table S2). The interventional study by Bertsche et al. [20] investigated drug-drug interactions (DDIs) and DDI-related adverse drug events in intensive care patients with a CDSS (Table 3). The content of the CDSS was developed by an interdisciplinary team with systematic literature searches. Randomization and blinding were not stated in the article; therefore, selection bias cannot be excluded.

Tisdale et al. [36] investigated the effectiveness of a CDSS including a risk score [12], for reducing the risk of QT prolongation (Table 3). The pre-intervention group and the implementation group differed significantly in some respects producing a risk of selection bias. Both the CDSS [36] and the included validated risk score [12] were developed in cardiac care units, limiting the external validity. Another limitation was the alert fatigue, as the override rate was $82 \%$. Of all alerts triggered, $13 \%$ resulted in additional 


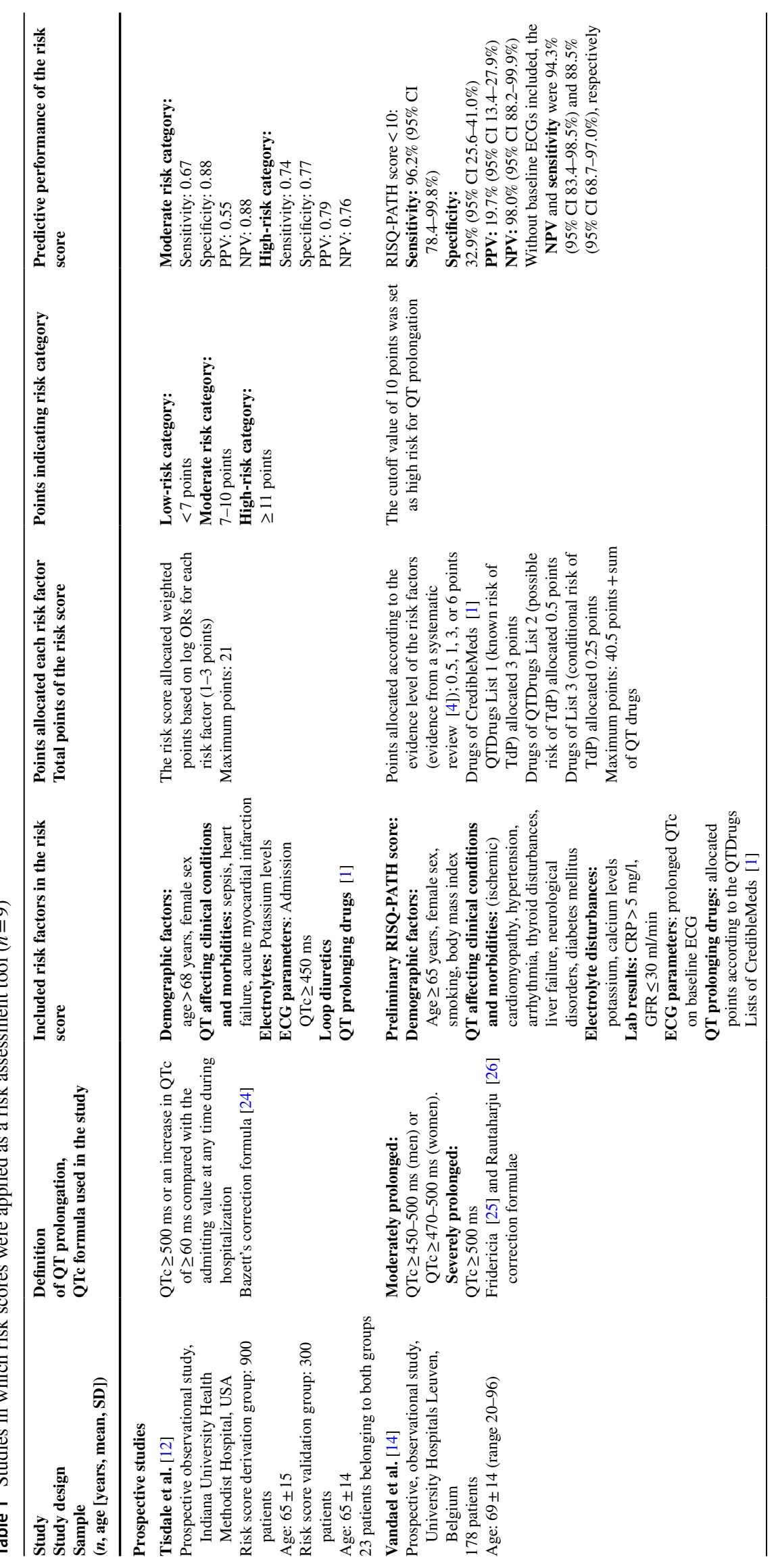




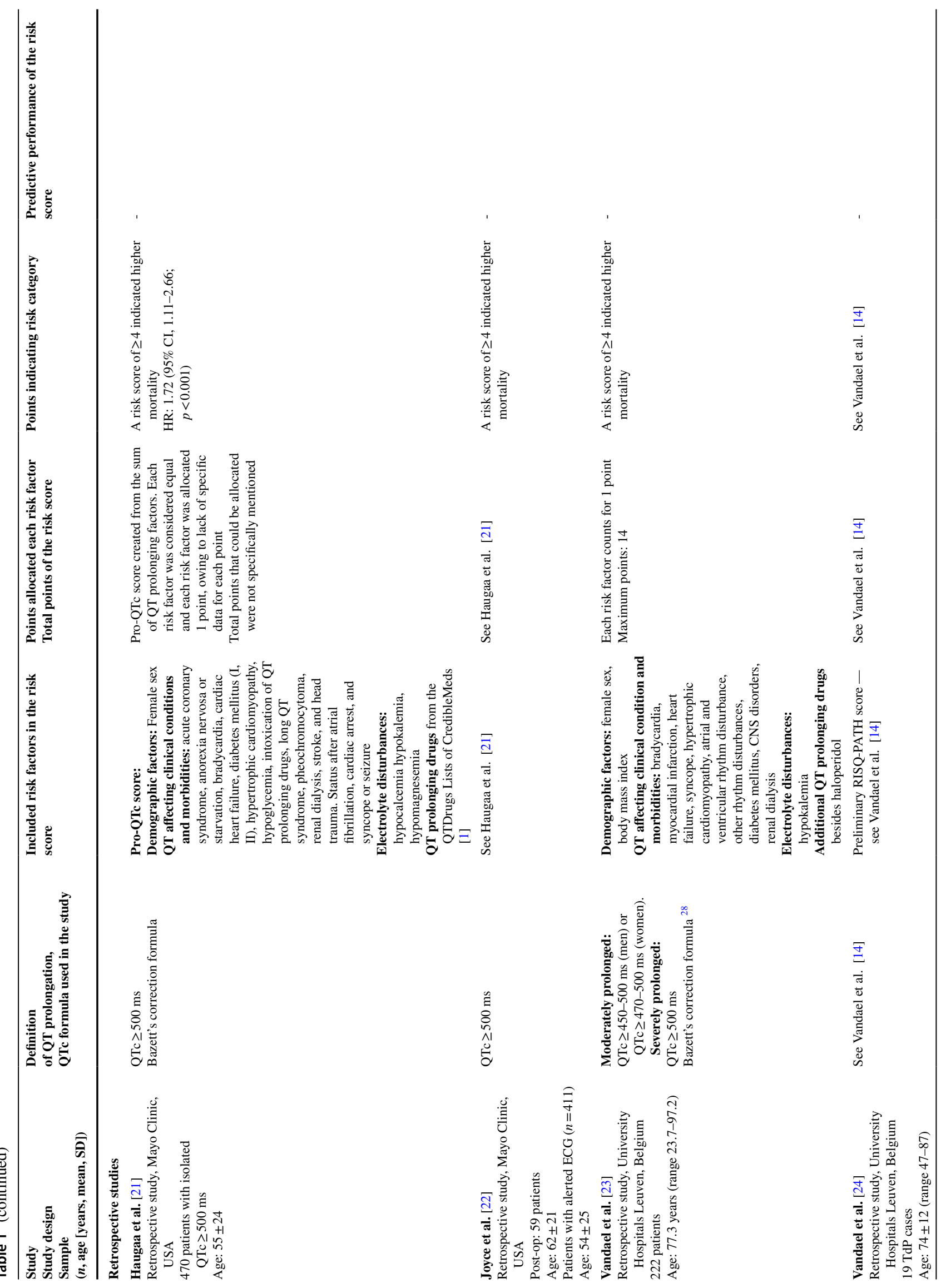




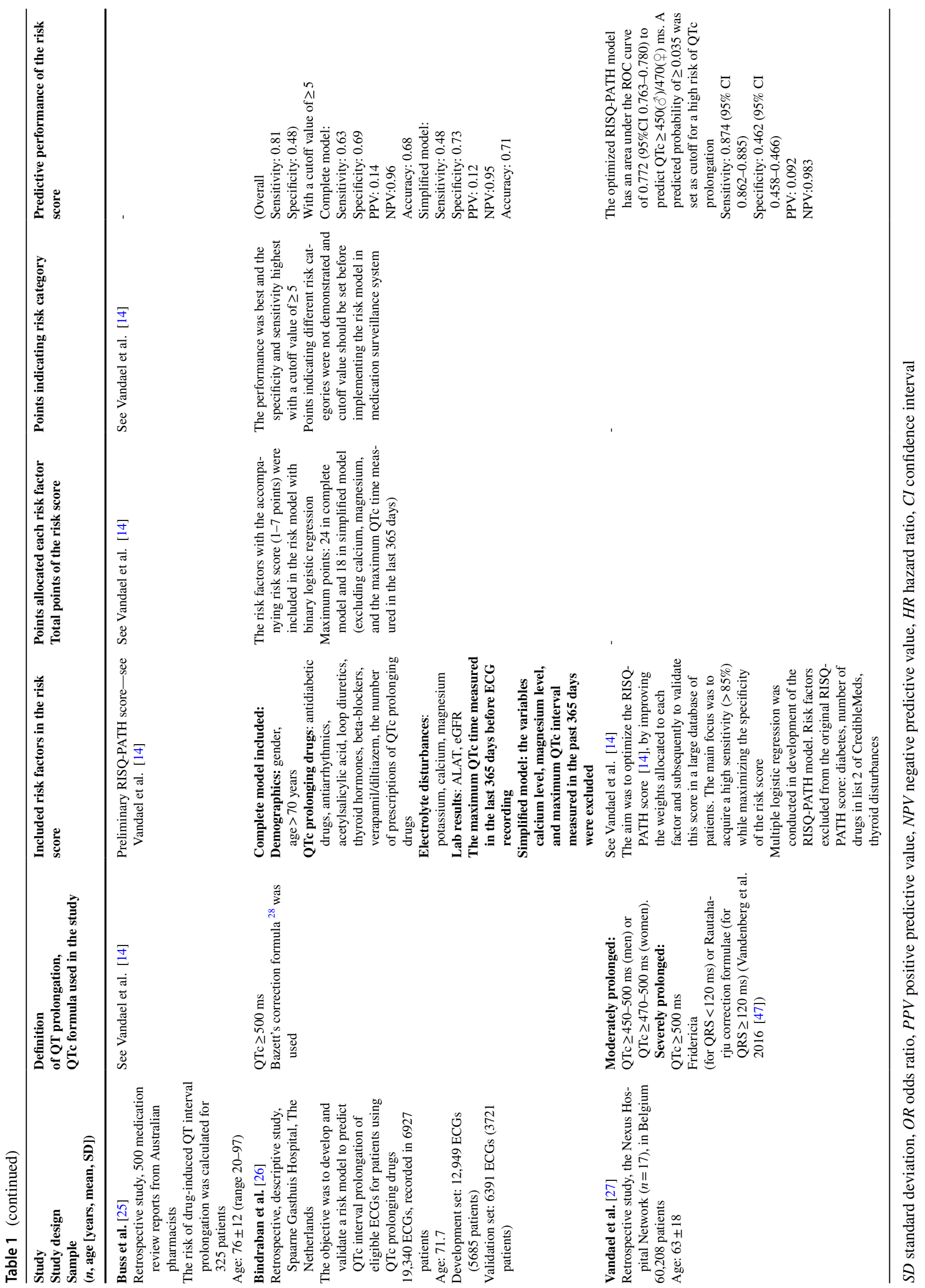




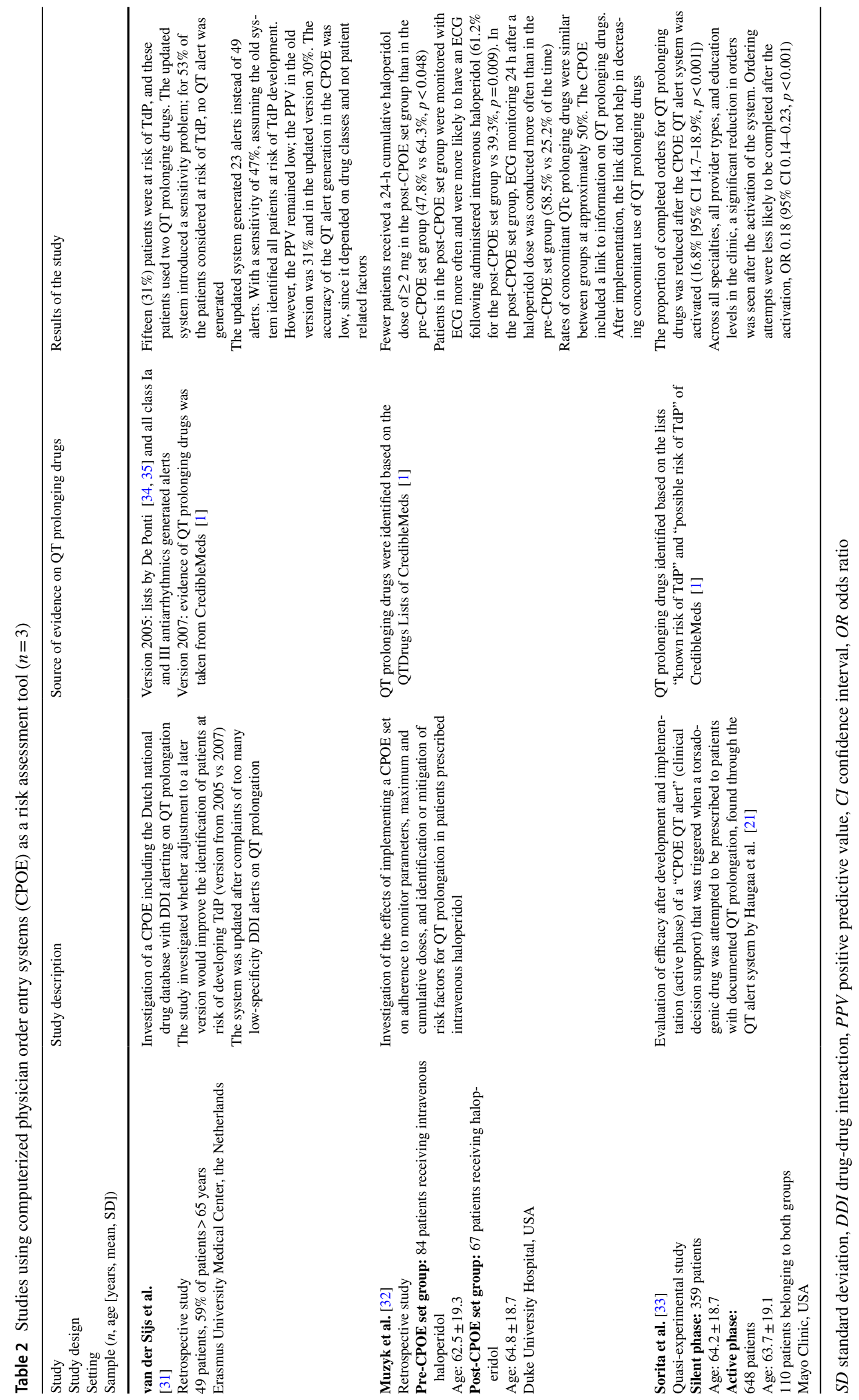




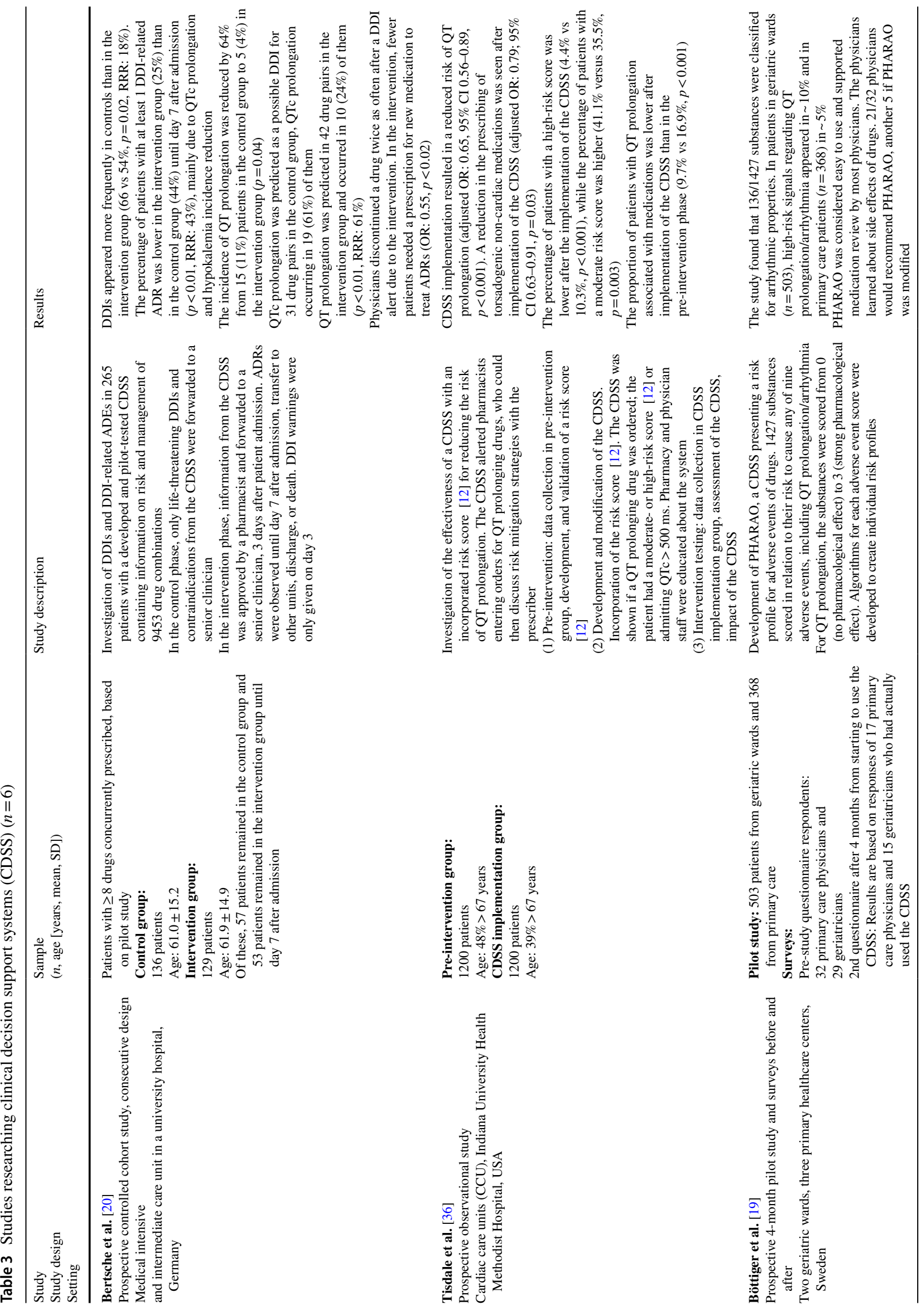




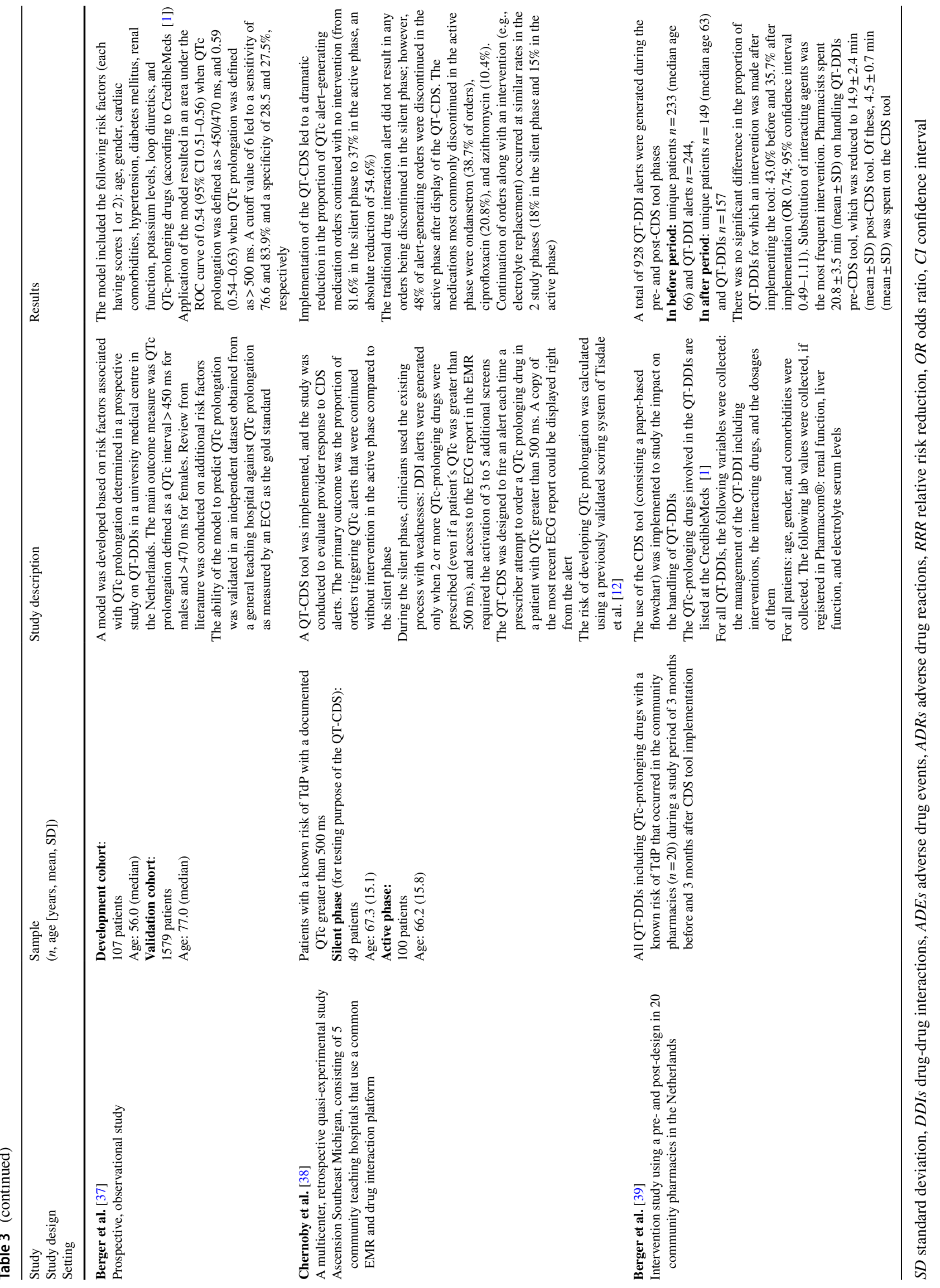


patient monitoring and $18 \%$ resulted in discontinued mediation orders.

Böttiger et al. [19] described the development of PHARAO (Pharmacological Risk Assessment Online), a CDSS presenting a risk profile for adverse events associated with combined effects of concomitantly used drugs (Table 3). A multidisciplinary team searched pharmacological handbooks, summaries of product characteristics, evaluations from European Medicines Agency, and articles through PubMed for evaluation and scoring of 1427 substances. PHARAO did not use pop-ups to alert physicians. To decrease alert fatigue, a restrictive approach was taken to what drug pairs would be included.

Berger et al. [37] developed a tool based on risk factors associated with QTc prolongation determined in a prospective study on QT-DDIs in a university medical center in the Netherlands. The main outcome measure was QTc prolongation defined as a QTc interval $>450 \mathrm{~ms}$ for males and $>470 \mathrm{~ms}$ for females. Review from literature was conducted on additional risk factors.

Chernoby et al. [38] conducted a retrospective study to evaluate provider response to CDS alert. The tool used the risk score by Tisdale et al. [12]. The primary outcome was the proportion of orders triggering QTc alerts that were continued without intervention in the active phase compared to the silent phase. The proportion reduced $55 \%$.

Berger et al. [39] studied the use of the CDS tool (consisting a paper-based flowchart) to assess the risk of QT drug-drug interactions in community pharmacies in the Netherlands. All QT-DDIs that occurred during a pre- and post-CDS tool period of 3 months were included. The QTcprolonging drugs involved in the QT-DDIs are listed at the CredibleMeds. Pharmacist intervened in $43.0 \%$ and $37.5 \%$ of the QT-DDIs pre- and post-CDS tool.

\section{Risk assessment tools focusing on ECG parameters $(n=3)$}

The QT nomogram [40], the $1 / 2$ RR rule [43], and the T-wave analysis software [44] were developed in retrospective studies. The RATs [40, 43, 44] focused on ECG parameters.
Chan et al. [40] developed a QT nomogram, based on a cloud diagram [41], for assessing the risk of TdP in a retrospective case-controlled study. Cases of drug-induced TdP $(n=130)$ were found in a systematic review (329 full-text articles reviewed) [40]. Controls $(n=318)$ were patients overdosing on non-cardiotoxic drugs, obtained from a previous study [42]. The systematic review included in the retrospective case-controlled study lacked description of included studies and assessment of risk of bias [40].

QT interval and heart rate combinations of cases and controls were plotted with the QT nomogram [40]. For comparison, two curves were plotted corresponding to Bazett's correction formula [28] at QTc values of $440 \mathrm{~ms}$ (medium-risk value) and $500 \mathrm{~ms}$ (high-risk value). The validity of some case points of tachycardia was questionable. The sensitivity and the specificity of the QT nomogram and Bazett's QTc [28] can be seen compared to the $1 / 2$ RR rule [43] and Fridericia's QTc [29] in Table 4.

The $1 / 2 \mathrm{RR}$ rule defines an abnormal QT interval as a QT greater than half of the RR interval on an ECG and does not require heart rate correction [43]. Berling and Isbister [43] conducted a study for determination of the sensitivity and specificity and comparison of the $1 / 2 \mathrm{RR}$ rule with other methods (Table 4 ). The obtainment of TdP cases $(n=129)$ and controls $(n=316)$ for their study from the literature was described by Chan et al. [40]. Additionally, the study by Berling and Isbister [43] calculated the agreement in eight different sample sets of QT-heart rate pairs from psychotropic medication overdoses. The $1 / 2 \mathrm{RR}$ rule [43] and the compared QT nomogram [40] both had poor negative and positive agreement, of which the latter was worse [43]. The $1 / 2$ RR rule misclassified patients without QT prolongation. Data was collected prospectively [43]. The sensitivity and specificity may be biased for all methods in Table 4 since the cases of TdP were taken from the literature.

Sugrue et al. developed a computer-based repolarization measurement tool to identify electrocardiographic predictors of torsadogenic risk [44]. The software provided extraction of information from automatic 12-lead ECGs. A retrospective $\mathrm{T}$ wave analysis of TdP cases and controls
Table 4 Sensitivity and specificity of the QT nomogram [40] and the $1 / 2$ RR rule [43] compared to QT correction formulae

\begin{tabular}{lll}
\hline Method & Sensitivity, \% (95\% CI) & Specificity, \% (95\% CI) \\
\hline QT nomogram [33] & $96.9(93.9-99.9)$ & $98.7(96.8-100)$ \\
$\begin{array}{l}\text { QT nomogram (cases with heart } \\
\text { rate }>104 \text { bpm excluded) [33] }\end{array}$ & $98.3(96.1-100)$ & $99.3(97.8-100)$ \\
1/2 RR rule [36] & $87.6(80.4-92.5)$ & $52.9(47.2-58.4)$ \\
1/2 RR rule $\geq 60$ bpm [36] & $100(94.6-100)$ & $49.7(43.8-55.5)$ \\
Bazett's QTc $=440 \mathrm{~ms}[33]$ & $98.5(96.4-100)$ & $66.7(58.6-74.7)$ \\
Bazett's QTc $=500 \mathrm{~ms}[33]$ & $93.8(89.6-98.0)$ & $97.2(94.3-100)$ \\
Fridericia's QTc $>500 \mathrm{~ms}[36]$ & $82.2(75.6-88.8)$ & $100(100-100)$ \\
\hline
\end{tabular}


using prescribed dofetilide and sotalol was conducted in Mayo Clinic, USA. The sample size was small $\left(n_{\text {cases }}=13\right.$, $n_{\text {controls }}=26$ ). There is potential for confounding by certain clinical factors that differed between the groups, as only sex and age were matched (within 2 years). QTc interval discriminated TdP cases from controls in $79 \%$. The sensitivity and specificity for QTc (Bazett's) alone were $88.1 \%$ and $72.0 \%$, respectively. The positive and negative predictive values were $85.0 \%$ and $76.9 \%$, respectively. The sensitivity and specificity in predicting TdP was $79.7 \%$ and $46.0 \%$, respectively, for the $\mathrm{T}$ wave right slope. The positive and negative predictive values were $58.9 \%$ and $70.0 \%$, respectively. Adding the $\mathrm{T}$ wave right slope in the analysis with QTc, the discrimination increased to $88 \%$.

\section{Discussion}

To our knowledge, this is the first systematic review that summarizes and compares tools for assessing safety risks of patients using QT prolonging drugs. Nine RATs were found of which those applying risk scores were most common, although QT risk assessment was also integrated in some CDSS and CPOE systems. Most of the risk assessment methods were used in inpatient care primarily by physicians and pharmacists. Many of the methods used the QTDrugs Lists of CredibleMeds [1] for identification of QT prolonging drugs. In addition to drugs associated with QT prolongation and TdP, the risk scores considered a wide range of non-pharmacological risk factors [12, 14, 21-27].

The risk scores [12, 14, 21-27] had similarities regarding the design of the risk score and included risk factors. A strength of the RISQ-PATH score [14, 24] was that it included a high number of risk factors and evidence of the included risk factors was assessed in a previously conducted systematic review [4]. Points were allocated according to evidence level [14]. The risk score by Tisdale et al. [12] allocated weighted points based on log odds ratios for each risk factor. As there is lower evidence of certain risk factors and certain drugs associated with QT prolongation and $\mathrm{TdP}$, it is sensible to allocate fewer points to these variables or exclude them completely from the RAT for better predictive performance. The risk score by Tisdale et al. [12] had good predictive performance and was also included in a CDSS [36]. The RISQ-PATH score had high sensitivity and negative predictive value [14]. The risk scores still need to be validated in prospective studies applying various patient groups and larger sample sizes.

The inclusion of patient-specific risk factors for QT prolongation and TdP in CPOE systems and CDSS varied [19, 20,31-33, 36]. CPOE systems and CDSS could be further developed to minimize alert fatigue [45]. In two included studies, alerts related to the risk for QT prolongation were overridden to a high degree $[33,36]$. Two of the studies indicated a reduction in the orders for QT prolonging drugs because of the alerts, but patient outcomes such as TdP were not measured [33, 38]. Böttiger et al. [19] restricted the drug pairs that were included in the CDSS as well as avoided popup alerts to avoid a high override rate.

These findings indicate the importance of designing CPOE systems and CDSS with increased sensitivity and specificity and to evaluate them regarding unnecessary generated signals. However, restricting drug pairs seems not to be the only solution to better performance of the RATs. In the updated version of the CPOE in the study by van der Sijs et al. [31], several drugs generating QT alerts were deleted, but the sensitivity problem of the CPOE remained. On the other hand, the RATs including other patient-specific risk factors have been found to excel in outcomes [12, 14, 33, 36]. Thus, further research is needed to optimize the tools for identifying clinically significant risks and patients at risk for QT prolongation and TdP. Future research should also be extended to cover the evaluation of impact of these tools on patient outcomes.

QT correction formulae were not assessed as RATs in our systematic review, but they were mentioned for studies using a correction formula. QT correction formulae have been previously studied [46, 47]. Many of the studies included in our systematic review used Bazett's correction formula [12, 19, 21, 23, 26, 28, 32, 36, 38, 40, 43, 44]. Bazett's correction formula has a problem with undercorrection and overcorrection of the QT interval [45]. Fridericia's correction formula seems to have better performance [47]. This formula was used in five of the included studies [14, 24, 27, 37, 43].

The QT nomogram performed better than Bazett's correction formula [40]. However, the QT nomogram [40], the $1 / 2$ RR rule [43], and the $T$ wave analysis tool [44] did not include other patient-specific risk factors than ECG parameters. The QTc is an indicator of risk of TdP, but it does not identify all risks $[6,11,12]$. If these RATs are used, patient-specific risk factors need to be assessed along with other RATs or based on clinical judgment. A survey from 2005 showed that the majority of the healthcare practitioners responding to the questionnaire could not correctly identify factors and drugs that may prolong the QT interval [49]. The T wave analysis tool [44] has potential as a RAT, but if used without considering QTc, it had lower predictive performance [44]. Further studies are needed of the QT nomogram [40] and the T wave analysis tool [44] that apply various patient groups and larger sample sizes, as well as other QT prolonging drugs.

Overall, the RATs may increase safety of patients at risk of QT prolongation or TdP as the studies either showed that a wide range of patient-specific risk factors were considered: ordering of QT prolonging drugs decreased, monitoring of patients increased, advice was given, or healthcare providers 
were trained about risks associated with QT prolongation. As older adults have an increased risk for QT prolongation and TdP due to the presence of multiple risk factors [6, 7], RATs, considering a wide range of patient-specific risk factors, may be useful when contemplating the use of QT prolonging drugs with these patients. Based on this systematic review, various RATs may be used in combination, e.g., risk scores for QT prolongation and TdP may be incorporated into CDSS and CPOE systems for easier access and risk assessment. Using RATs in combination may also identify high-risk patients from low risk patients and may reduce unnecessary alerts.

This systematic review may assist in the decision to select and use RATs for QT prolonging pharmacotherapy. The included RATs could be further developed to fit different health information systems. There is potential for the RATs to be adapted to outpatient settings and furthermore assist other healthcare providers other than physicians, e.g., community pharmacists [19, 39]. However, no guidelines for managing risks of QT prolongation in primary care or community pharmacies exist and more training on the topic is needed to adopt the tools in clinical practice $[48,50]$.

The review was conducted according to the PRISMA guidelines $[15,16]$. In the process, the GRADE approach was considered [18]. In the search process, the included studies found in PubMed were searched for additional studies. Since only duplicates were found in the search of the Scopus database, another database could possibly have been searched. Most of those included were observational studies, which are more prone to bias because of their design [51]. However, since TdP occurs rarely [40, 52], an observational study design can be useful in finding these events. Clinical validation of QT-RATs with RCT studies is needed, as our systematic review shows that studies are mainly observational or quasi-experimental studies and RCT studies were not found. A comprehensive bias assessment using a bias assessment tool would be useful in future studies, as it was not conducted in this study. Risk of bias assessment was conducted only by the authors, considering bias assessment presented in the included studies.

\section{Conclusions}

Most of the RATs for QT prolonging pharmacotherapy give a comprehensive overview on patient-specific risks of QT prolongation and $\mathrm{TdP}$ and reduce modifiable risk factors and actual events. There is potential for the RATs to be adapted to different health information systems in inpatient and outpatient settings. Studies on outcomes of using individual RATs and combining various tools and clinical validation of QT-RATs with RCT studies are needed.
Supplementary Information The online version contains supplementary material available at https://doi.org/10.1007/s00228-022-03285-3.

Acknowledgements Information Specialist Katri Larmo, Medical Library, University of Helsinki, Finland, is acknowledged for assisting with the literature search and search terms. Juha Puustinen received Grant VTR from Satakunta Hospital District and Varsinais-Suomi Hospital District to support analysis and reporting of this study.

Author contribution All of the authors (Skullbacka, Toivo, Airaksinen, and Puustinen) conceived of or designed study. Skullbacka, Toivo, and Airaksinen conducted the screening and selection of the studies. All of the authors participated in the data extraction and risk of bias assessment. All of the authors participated in drafting the manuscript and approved the final version to be published.

Funding Open Access funding provided by University of Helsinki including Helsinki University Central Hospital.

Data availability All data generated or analyzed during this study are presented in this manuscript.

Code availability Not applicable.

\section{Declarations}

Competing interests The authors declare no competing interests.

Open Access This article is licensed under a Creative Commons Attribution 4.0 International License, which permits use, sharing, adaptation, distribution and reproduction in any medium or format, as long as you give appropriate credit to the original author(s) and the source, provide a link to the Creative Commons licence, and indicate if changes were made. The images or other third party material in this article are included in the article's Creative Commons licence, unless indicated otherwise in a credit line to the material. If material is not included in the article's Creative Commons licence and your intended use is not permitted by statutory regulation or exceeds the permitted use, you will need to obtain permission directly from the copyright holder. To view a copy of this licence, visit http://creativecommons.org/licenses/by/4.0/.

\section{References}

1. Woosley RL, Heise CW, Romero KA (2018) QTdrugs List, January 2018, AZCERT, Inc. 1822 Innovation Park Dr., Oro Valley, AZ 85755. Available at: www.crediblemeds.org Accessed January 24, 2018

2. Thomas SHL, Behr ER (2016) Pharmacological treatment of acquired QT prolongation and torsades de pointes. Br J Clin Pharmacol 81(3):420-427

3. Schwartz PJ, Woosley RL (2016) Predicting the unpredictable. Drug-induced QT prolongation and torsades de pointes. J Am Coll Cardiol 67:1639-1650

4. Vandael E, Vandenberk B, Vandenberghe J et al (2017) Risk factors for QTc-prolongation: systematic review of the evidence. Int J Clin Pharm 39(1):16-25

5. Laksman Z, Momciu B, Seong YW et al (2015) A detailed description and assessment of outcomes of patients with hospital recorded QTc prolongation. Am J Cardiol 115(7):907-911 
6. Drew BJ, Ackerman MJ, Funk M et al (2010) Prevention of Torsade de Pointes in hospital settings: a scientific statement from the American Heart Association and the American College of Cardiology Foundation. Circulation 121(8):1047-1060

7. Moreno-Gutiérrez PA, Gaviria-Mendoza A, Montoya Cañón M et al (2016) High prevalence of risk factors in elderly patients using drugs associated with acquired torsades de pointes chronically in Colombia. Br J Clin Pharmacol 82:504-511

8. Curtis LH, Østbye T, Sendersky V et al (2003) Prescription of QT-prolonging drugs in a cohort of about 5 million outpatients. Am J Med 114:135-141

9. Tay K, Ewald MB, Bourgeois FT (2014) Use of QT-prolonging medications in US emergency departments, 1995-2009. Pharmacoepidemiol Drug Saf 23(1):9-17

10. Schächtele S, Tümena T, Gaßmann KG et al (2016) Co-prescription of QT-interval prolonging drugs: an analysis in a large cohort of geriatric patients. PLoS One 11(5):e0155649.

11. Roden DM (2004) Drug-induced prolongation of the QT interval. N Engl J Med 350(10):1013-1022

12. Tisdale JE, Jaynes HA, Kingery JR et al (2013) Development and validation of a risk score to predict QT interval prolongation in hospitalized patients. Circ Cardiovasc Qual Outcomes 6(4):479-487

13. Malik M (2001) Problems of heart rate correction in assessment of drug-induced QT interval prolongation. J Cardiovasc Electrophysiol 12(4):411-420

14. Vandael E, Vandenberk B, Vandenberghe J et al (2017) Development of a risk score for QTc-prolongation: the RISQ-PATH study. Int J Clin Pharm 39(2):424-432

15. Moher D, Liberati A, Tetzlaff J et al (2009) Preferred reporting items for systematic reviews and meta-analyses: the PRISMA statement. PLoS Med 6(7):e1000097

16. Page MJ, McKenzie JE, Bossuyt PM, Boutron I, Hoffmann TC, Mulrow CD et al (2021) The PRISMA 2020 statement: an updated guideline for reporting systematic reviews. BMJ 372:n71. https://doi.org/10.1136/bmj.n71

17. World Health Organization (2017) WHO: proposed working definition of an older person in Africa for the MDS Project. (online) Available at: www.who.int. Accessed October 8, 2017

18. Guyatt GH, Oxman AD, Vist GE et al (2008) GRADE: an emerging consensus on rating quality of evidence and strength of recommendations. BMJ 336(7650):924-926

19. Böttiger Y, Laine K, Korhonen T et al (2017) Development and pilot testing of PHARAO- a decision support system for pharmacological risk assessment in the elderly. Eur J Clin Pharmacol 1-7

20. Bertsche T, Pfaff J, Schiller P et al (2010) Prevention of adverse drug reactions in intensive care patients by personal intervention based on an electronic clinical decision support system. Intensive Care Med 36(4):665-672

21. Haugaa KH, Bos JM, Tarrell RF (2013) Institution-wide QT alert system identifies patients with a high risk of mortality. Mayo Clin Proc 88(4):315-325

22. Joyce DD, Bos JM, Haugaa KH et al (2015) Frequency and cause of transient QT prolongation after surgery. Am J Cardiol 116(10):1605-1609

23. Vandael E, Vandenberk B, Vandenberghe J et al (2016) Risk management of QTc-prolongation in patients receiving haloperidol: an epidemiological study in a University hospital in Belgium. Int J Clin Pharm 38(2):310-320

24. Vandael E, Vandenberk B, Vandenberghe J et al (2017) Incidence of Torsade de Pointes in a tertiary hospital population. Int J Cardiol 243:511-515

25. Buss VH, Lee K, Naunton M et al (2018) Identification of patients at-risk of QT interval prolongation during medication reviews: a missed opportunity? J Clin Med 7:533
26. Bindraban AN, Rolvink J, Berger FA et al (2018) Development of a risk model for predicting QTc interval prolongation in patients using QTc-prolonging drugs. Int J Clin Pharm 40(5):1372-1379. https://doi.org/10.1007/s11096-018-0692-y

27. Vandael E, Vandenberk B, Vandenberghe J et al (2018) A smart algorithm for the prevention and risk management of QTc prolongation based on the optimized RISQ-PATH model. Br J Clin Pharmacol 84(12):2824-2835. https://doi.org/10.1111/bcp. 13740

28. Bazett $H$ (1920) An analysis of the time relationship of electrocardiograms. Heart 7:353-370

29. Fridericia LS (1920) Die Systolendauer im elektrokardiogramm bei normalen menschen und bei herzkranken. Acta Med Scand 53:489

30. Rautaharju PM, Zhang ZM, Prineas R, Heiss G (2004) Assessment of prolonged QT and JT intervals in ventricular conduction defects. Am J Cardiol 93:1017-1021

31. van der Sijs H, Kowlesar R, Aarts J et al (2009) Unintended consequences of reducing QT-alert overload in a computerized physician order entry system. Eur J Clin Pharmacol 65(9):919-925

32. Muzyk A, Rivelli S, Jiang W et al (2012) A computerized physician order entry set designed to improve safety of intravenous haloperidol utilization. Drug Saf 35(9):725-731

33. Sorita A, Bos JM, Morlan BW et al (2015) Impact of clinical decision support preventing the use of QT-prolonging medications for patients at risk for torsade de pointes. J Am Med Inform Assoc 22:e21-e27

34. De Ponti F, Poluzzi E, Montanaro N (2000) QT-interval prolongation by non-cardiac drugs: lessons to be learned from recent experience. Eur J Clin Pharmacol 56(1):1-18

35. De Ponti F, Poluzzi E, Montanaro N (2001) Organising evidence on QT prolongation and occurrence of torsades de pointes with non-antiarrhythmic drugs: a call for consensus. Eur J Clin Pharmacol 57(3): 185-209

36. Tisdale JE, Jaynes HA, Kingery JR et al (2014) Effectiveness of a clinical decision support system for reducing the risk of QT interval prolongation in hospitalized patients. Circ Cardiovasc Qual Outcomes 7(3):381-390

37. Berger FA, van der Sijs H, Becker ML et al (2020) Development and validation of a tool to assess the risk of QT drug-drug interactions in clinical practice. BMC Med Inform Decis Mak 20: 171

38. Chernoby K, Lucey MF, Hartner CL, Dehoorne M, Edwin SB (2020) Impact of a clinical decision support tool targeting QT-prolonging medications. Am J Health Syst Pharm 77(Supplement_4):S111S117. https://doi.org/10.1093/ajhp/zxaa269

39. Berger FA, van der Sijs H, van Gelder T, van den Bemt PMLA (2021) The use of a clinical decision support tool to assess the risk of QT drug-drug interactions in community pharmacies. Ther Adv Drug Saf 12:2042098621996098

40. Chan A, Isbister GK, Kirkpatrick CMJ et al (2007) Drug-induced QT prolongation and torsades de pointes: evaluation of a QT nomogram. QJM 100(10):609-615

41. Fossa AA, Wisialowski T, Magnano A et al (2005) Dynamic beat-tobeat modeling of the QT-RR interval relationship: analysis of QT prolongation during alterations of autonomic state versus human ether a-go-go-related gene inhibition. J Pharmacol Exp Ther 312(1):1-11

42. Isbister GK, Bowe SJ, Dawson A et al (2004) Relative toxicity of selective serotonin reuptake inhibitors (SSRIs) in overdose. Clin Toxicol 42(3):277-285

43. Berling I, Isbister GK (2015) The half RR rule: a poor rule of thumb and not a risk assessment tool for QT interval prolongation. Acad Emerg Med 22(10):1139-1144

44. Sugrue A, Kremen V, Qiang B et al (2015) Electrocardiographic predictors of torsadogenic risk during dofetilide or sotalol initiation: utility of a novel T wave analysis program. Cardiovasc Drugs Ther 29(5):433-441 
45. van der Sijs H, Aarts J, Vulto A et al (2006) Overriding of drug safety alerts in computerized physician order entry. J Am Med Inform Assoc 13(2):138-147

46. Luo S, Michler K, Johnston P et al (2004) A comparison of commonly used QT correction formulae: the effect of heart rate on the QTc of normal ECGs. J Electrocardiol 37:81-90

47. Vandenberk B, Vandael E, Robyns T et al (2016) Which QT correction formulae to use for QT monitoring? J Am Heart Assoc 5(6): 003264

48. Toivo T, Dimitrow M, Puustinen J et al (2018) Coordinating resources for prospective medication risk management of older home care clients in primary care: procedure development and RCT study design for demonstrating its effectiveness. BMC Geriatr 18(74):1-10

49. Al-Khatib SM, Allen LaPointe NM, Kramer JM et al (2005) A survey of health care practitioners' knowledge of the QT interval. J Gen Intern Med 20:392-396
50. Vandael E, Verstuyft E, Leirs C et al (2018) An e-learning programme about the risk and management of QTc-prolongation in community pharmacies significantly improves pharmacists' (longterm) knowledge. Pharm Educ 18(1):119-131

51. Schünemann H, Jan Brożek J, Guyatt G et al (2018) GRADE handbook, 2013 (online). Available at: http://gdt.guidelinedevelopment. org/app/handbook/handbook.html. Accessed May 09, 2018.

52. Darpö B (2001) Spectrum of drugs prolonging QT interval and the incidence of torsades de pointes. Eur Heart J Suppl 3(Suppl $\mathrm{K}): \mathrm{K} 70-\mathrm{K} 80$

Publisher's Note Springer Nature remains neutral with regard to jurisdictional claims in published maps and institutional affiliations. 